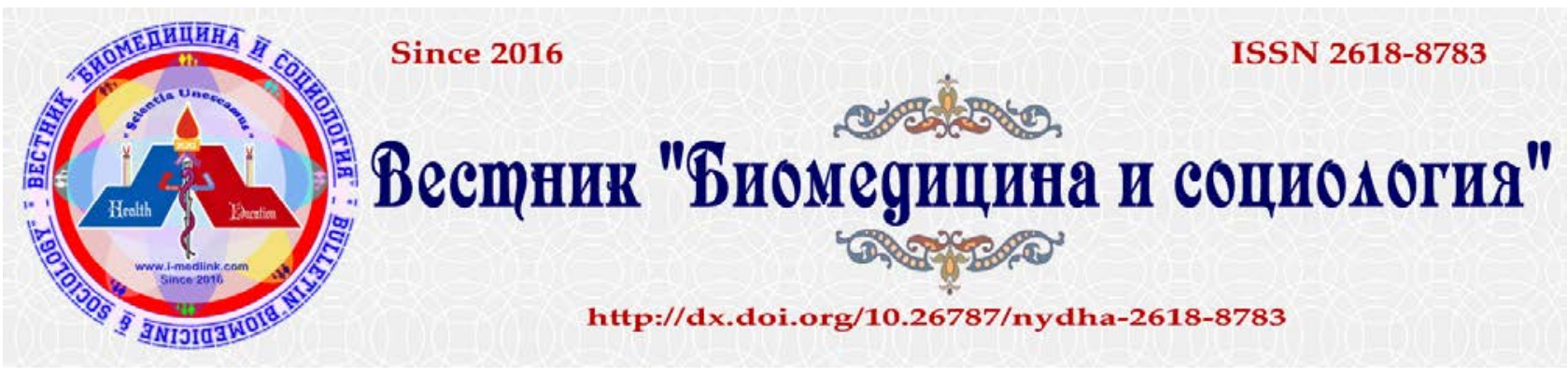

2018, том 3 [4]

http://dx.doi.org/10.26787/nydha-2618-8783-2018-3-4-23-25

\title{
THE EFFECT OF SMOKING ON THE MALE FACTOR OF INFERTILITY
}

Alsaed Laith W.M., Barkhudarov A.

RUDN University, Moscow, Russian Federation

Annotation. Cigarette smoking is widespread among the male population.

Objective: to identify the degree of influence of cigarette smoking on spermatogenesis.

Materials and Methods: 70 patients were examined, whose wife did not have a pregnancy in the course of 1 year of marriage. All men abused cigarette smoking.

Results: $60 \%$ of patients smoked 1 pack of cigarettes per day. Asthenozoospermia is the most common among them - 45 (64,2\%). Azoospermia was detected in $5(7,1 \%)$ patients who abused tobacco.

Findings: With prolonged smoking in the testicular tissue, an increased apoptosis of spermatogenic cells is observed.

Key words: male infertility, cigarette smoking, apoptosis.

Over the past decade, 52,6 million infertile couples have been registered in the world [5]. Male infertility occurs in $40-50 \%$ of cases [1].

Spermatogenesis is a complex process among fetal cells. During their development, spermatogenic cells undergo cycles of mitotic and meiotic division, with the subsequent formation of mature spermatozoa with a haploid set [3]. Spermatogenesis occurs under the control of specific genes of the developing gametes and is regulated by a combination of hormones, cytokines, growth factors, synthesized both in the organs of the reproductive system and outside it [4]. Therefore, a violation in any part of the regulation of spermatogenesis leads to the development of male infertility.

The most severe and critical form of impaired spermatogenesis is nonobstructive azoospermia [2, 4]. There are many causes leading to patospermia: both endogenous and exogenous. Cigarette smoking is widespread among the male population. Approximately 35\% of men of reproductive age in the United States smoke cigarettes and $45 \%$ of men in Eastern countries [5]. However, today there is a scant amount of research devoted to the effect of smoking on male reproductive function.

Objective: to identify the extent to which cigarette smoking affects spermatogenesis.

Materials and methods: 70 patients were examined, whose wife did not have a pregnancy in the course of 1 year of marriage. All men abused cigarette smoking. All patients had a normal karyotype.

We analyzed the following data: passport information, the nature of complaints, anamnestic, physical, laboratory. We paid special attention to the history: cigarette smoking (quantity per day).

Patients who used narcotic substances (including marijuana), who had varicocele, cancer, developmental abnormalities were excluded from the study.

Ejaculate values were assessed according to the World Health Organization protocol (WHO Laboratory Manual, 5th edition, Geneva, 2010).

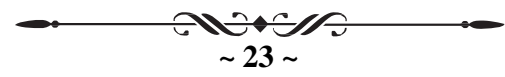

Вестник представлен в научной электронной библиотеке (НЭБ) - головном исполнителе проекта по созданию Российского индекса научного цитирования (РИНЦ) 
According to the testimony, we performed a biopsy of the testicle. Testicular tissue was subjected to histological examination. Using the method of indirect immunohistochemical analysis (IHH), we identified the protein apoptosis - caspasa-9.

Statistical processing of the material was carried out using spreadsheets «EXCEL» and the program «STATISTICA 6.0». The assessment of the reliability of differences between quantitative indicators was performed using the Mann-Whitney test. To compare the qualitative parameters, the Fisher exact test or $\chi 2$ was used. Differences were considered significant at $p<0,05$.

Results: All patients were between the ages of 24 and 46 . The average age of all patients was 32,3 \pm 6 years.

The majority of patients in the study group - 42 (60\%) smoked 1 pack of cigarettes per day (Figure 1). Less than 1 pack of cigarettes per day smoked 18 (17,1\%) men.

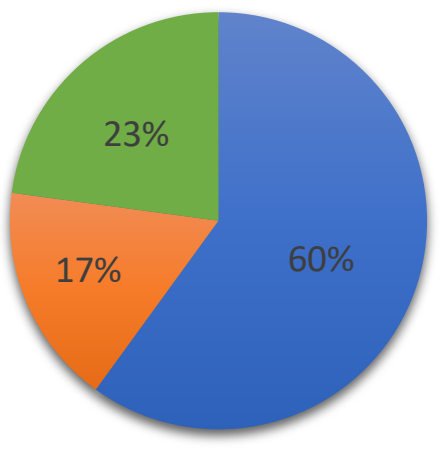

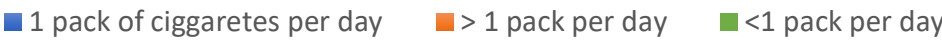

Figure 1. The distribution of patients by the number of cigarette smoking.

Indicators of spermograms in patients who smoked cigarettes are presented in Figure 2. In this category of patients, asthenozoospermia is the most common - 45 (64,2\%). Azoospermia was detected in 5 $(7,1 \%)$ patients who abused tobacco.

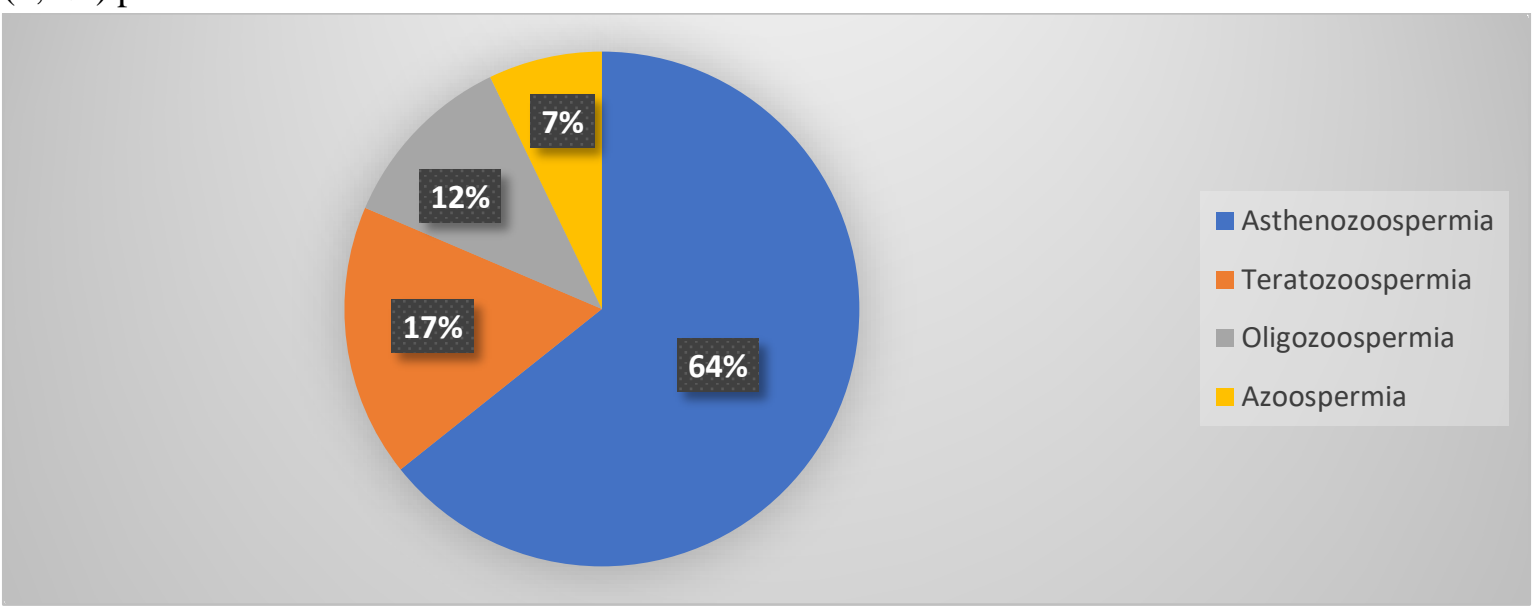

Figure 2. Sperm data in patients who smoked cigarettes.

The results of indirect immunohistochemical analysis (visualization of apoptosis protein - caspasa9) are presented in Figure 3. The largest proportion of IHC-positive spermatogonia in the seminiferous tubules in patients who smoked cigarettes was detected with a histological pattern of the focal variant of Sertoli cell syndrome - $12(17,1 \%)$.

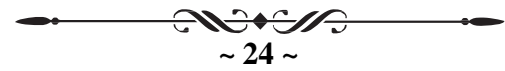




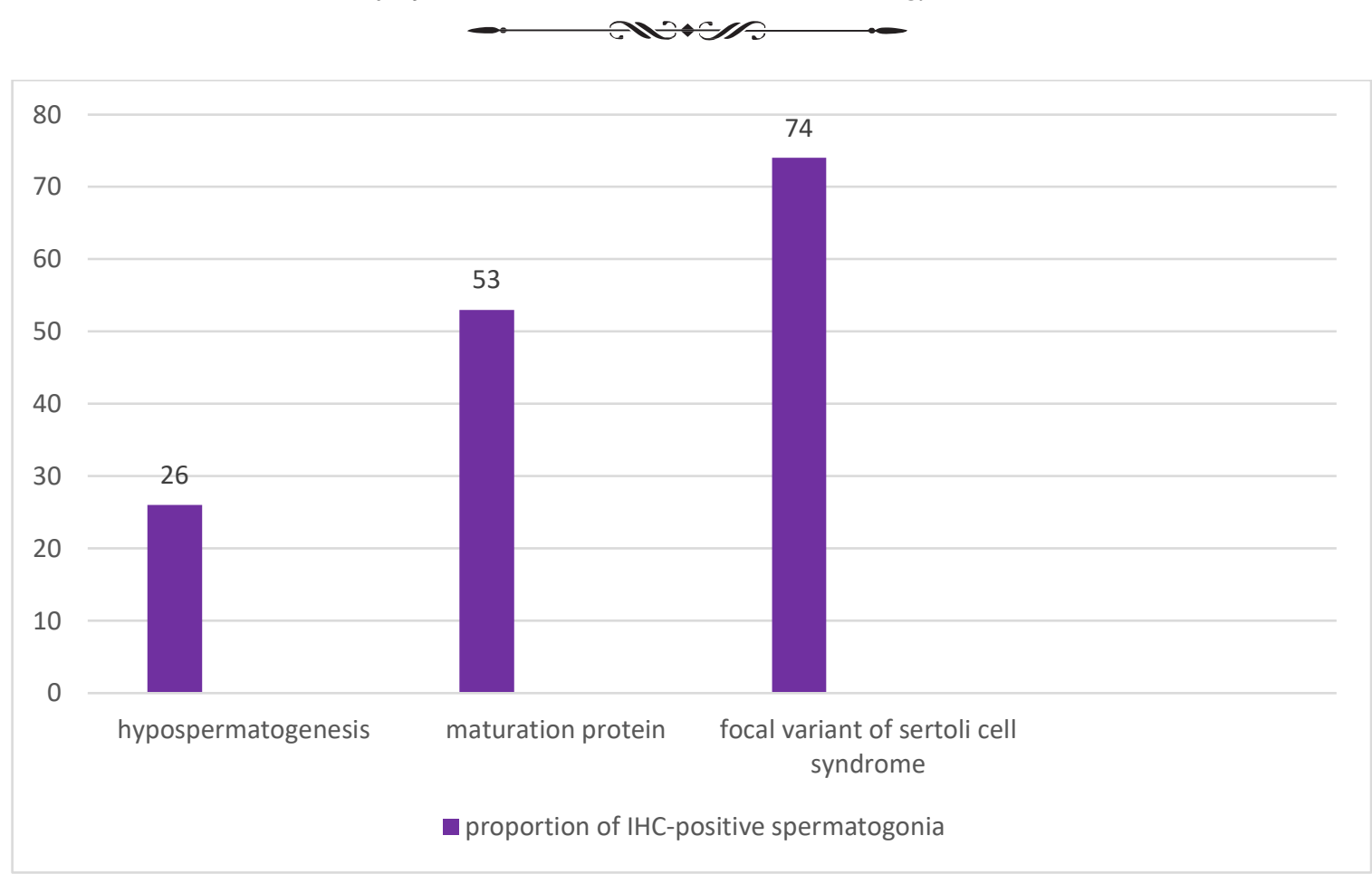

Figure 3. The proportion of IHC-positive spermatogonia in the seminiferous tubules in patients who smoked cigarettes.

Apoptosis is a regulated process of cell death. Excess proliferation of spermatogonia is normally regulated by selective apoptosis and lasts continuously throughout life. The role of caspases in sperm differentiation and testicular maturity is of considerable interest. Smoking causes peripheral vasoconstriction, which leads to a decrease in the oxygen content in the tissues. With prolonged smoking, testicular tissue and spermatogenic cells experience chronic hypoxia. And, chronic hypoxia contributes to the implementation of the trigger signal to apoptosis.

Conclusion: cigarette smoking causes an increase in apoptosis and contributes significantly to the violation of spermatogenesis. Without deep knowledge of the factors that affect the testicle and have a positive or negative effect on its reproductive function, it is impossible to solve the problems of diagnosing male infertility.

\section{REFERENCES}

1. Gromov A.I., Bujlov V.M. Radiation diagnostics and therapy in urology: national manual / M.: GOEHTARMedia, 2011. 544p.

2. Kulchenko N.G. Oxidative stress in the development of non-obstructive azoospermia // Difficult patient. 2017;15(4-5):44-46.

3. Kulchenko N.G. Inflammation and infertility. What is common? // RUDN Journal of Medicine, 2017, 21 (4), 402-407.

4. Kulchenko N.G. Prediction of success in assisted reproductive technology with the help of morphology of the testis. Research'n Practical Medicine Journal. 2018;5(4):18-25.

5. Kiter G., Başer S., Akdag B., Ekinci A., Ünal N., Öztürk E. The characteristics of smoking habit among patients evaluated at our outpatient clinic. Tuberk Toraks. 2008;56(1):30-6.

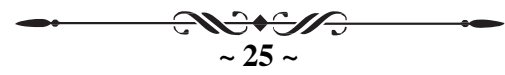

